\title{
Correction to: A Survey of Deep Learning Applied to Story Generation
}

Chenglong Hou, Chensong Zhou, Kun Zhou, Jinan Sun, and Sisi Xuanyuan

\section{Correction to:}

Chapter "A Survey of Deep Learning Applied to Story

Generation" in: M. Qiu (Ed.): Smart Computing

and Communication, LNCS 11910,

https://doi.org/10.1007/978-3-030-34139-8_1

In the version of this paper that was originally published, the last name of the author

Sisi Xuanyuan was misspelt. This has been corrected. 